\title{
Effects of the COVID-19 lockdown on musculoskeletal pain, physical activity, and work environment in Estonian office workers transitioning to working from home
}

\author{
Martin Argus* and Mati Pääsuke \\ Institute of Sport Sciences and Physiotherapy, University of Tartu, Estonia
}

Received 12 January 2021

Accepted 5 April 2021

\begin{abstract}
.
BACKGROUND: COVID-19 lockdown caused a sudden change in the work culture and environment.

OBJECTIVE: The aim of this study was to evaluate the impact of COVID-19 lockdown caused changes in musculoskeletal pain (MSP), physical activity (PA), workplace properties, and their in-between relationships among office workers.

METHODS: A total of 161 office workers (64.6\% female) with a mean age of $38.2 \pm 9.5$ years participated. The study was conducted as an online form. Baecke Physical Activity Questionnaire, NORDIC Questionnaire, and questions about the work environment were used. The participants were asked to describe the current situation and retrospectively the situation 3 months before.

RESULTS: We found no significant differences in the prevalence of MSP or between the mean number of body regions suffering from MSP before and during the lockdown. During the lockdown, a significant $(p<0.001)$ reduction in total PA and sport-related PA and a significant $(p<0.001)$ increase in work-related PA was noted. A significant drop in both workplace comfort rating $(p<0.05)$ and workplace ergonomics rating $(p<0.001)$ was suggested during the lockdown. Our data also suggested several individual factors influencing the MSP among office workers during the lockdown.

CONCLUSIONS: Maintaining habitual physical activity level and preparing a more comfortable and ergonomic workspace can play a role in a healthier transition to working from home.
\end{abstract}

Keywords: COVID-19, musculoskeletal pain, office workers, physical activity, work environment

\section{Introduction}

The coronavirus (COVID-19) pandemic was first announced in Wuhan, China in December 2019, and on March 11, 2020, the World Health Organization (WHO) declared a global pandemic [1]. The

*Address for correspondence: Martin Argus, MSc; E-mail: martin.argus@ut.ee. first case of coronavirus in Estonia was discovered on February 26, 2020, and on March 12, 2020, the government of Estonia declared an emergency situation. The main methods for reducing the spread of the virus were implementing social distancing rules, restricting travel, indoor and outdoor gatherings, and services including physical contact. The emergency situation ended on May 17, 2020, lasting a total of 66 days [2]. The emergency situation changed a lot about how people worked and moved. During the 
emergency situation in Estonia, the sport and fitness centers were closed, but people were allowed to exercise and move outdoors when social distancing rules were followed. Due to social distancing rules, office spaces were closed and workers had to work from home. Until the virus persists, many companies in Estonia continue to implement teleworking. It is predicted, that this changes how we work in the future, as in addition to reducing the chance of spreading viruses, remote work is proving to have benefits both on an organizational and individual level $[3,4]$.

Teleworking, also known as remote work, has been gaining popularity due to rapid advances in information technology and electronic equipment. There have been reported several individual benefits of telework, such as lower stress due to less time spent on commuting, better work output as a result of fewer interruptions, fewer sick days and depending on the organization, flexible schedule, and better work-family balance. Despite the benefits, there are several problems regarding teleworking and occupational health. The main concerns include excess working hours, workers might continue working when ill, ignore health-related problems, and not have adequate equipment $[5,6]$. Several psychosocial factors have also been reported, such as the feeling of isolation and possible conflict between work and family life [6]. In the context of COVID-19, the sudden shift to teleworking for an unknown time might have caused less attention to workplace safety and ergonomics [7]. Therefore this sudden change to teleworking might have an impact on musculoskeletal health.

The prevalence of musculoskeletal pain (MSP) is high among office workers $[9,10]$. MSP can have a significant negative impact on the quality of life, work efficiency, and work ability [10, 11]. During the 3month coronavirus lockdown in Turkey, increases in the prevalence of low-back pain in people who stayed home compared to those who kept going to the office were reported [12]. In Italy, Moretti and colleagues described a worsening of low-back pain and neck pain in the remote workers questioned [13], however, it is not known how teleworking affected the prevalence of MSP.

There have been documented several risk factors for developing MSP including individual factors and inadequate workplace equipment and ergonomics [8]. One of the individual factors, that could be influenced by the lockdown restrictions, is low physical activity (PA) $[14,15]$. PA and sedentary lifestyle is a known risk factor for poor overall health, premature mortality, and several types of disease, including musculoskeletal disorders [14, 16-18]. Increasing PA has proven several benefits for office workers, such as reducing work-related and general MSP $[19,20]$ better work ability [21], and better mental health [22]. Since it is reported, that quality of life, well-being, and mental health problems have worsened during COVID-19 [23], PA might play an important role in how we prevent both physical and mental health issues and complex biopsychosocial issues like MSP in the future. It is known, that the restrictions during the lockdown period had a negative effect on the overall PA of adults in Greece [24], but we are uncertain how exactly people with physically inactive work, such as office workers, were affected. It is also unknown how such sudden changes in PA on an individual level affect MSP.

Another factor associated with MSP is workplace ergonomics. There are several theories about the mechanisms of pain pathogenesis in the case of cognitive work with low physical demands, but the exact mechanism is still unclear [25-27]. Still, an association between workplace ergonomics and the prevalence of MSP has been noticed widely [8, 28]. Since there is some evidence on the effect of comfortable work equipment on MSP [29, 30], the sudden change to working from home might influence workrelated MSP, as we hypothesize that most homes do not have ergonomic workstations.

The aim of this study was to evaluate the COVID19 lockdown caused changes in MSP, PA, workplace properties, and their in-between relationships among office workers. Considering the recent research about MSP during COVID-19 [12, 13, 31], we hypothesize an increase in the prevalence of MSP among office workers during the lockdown in Estonia. We also hypothesize, that because of the shift to remote work and closure of sports facilities, office workers report higher work-time PA, lower sport-related PA compensating with higher leisure-time PA during the lockdown period. Due to the sudden transition to working from home, we hypothesize lower workplace comfort and ergonomics scores during the lockdown period.

\section{Materials and methods}

\subsection{Subjects}

A total of 161 office workers (64.6\% females) with an age range of $20-59$ years (mean $38.17 \pm 9.52$ 
years) participated in this study. The mean work experience as an office worker was $13.07 \pm 8.59$ years. The inclusion criteria were job described as office work, working with a computer at least 6 hours per day, age 18-60 years. The exclusion criteria were chronic neurological, orthopedic, metabolic, or inflammatory conditions, and legally-designated disability. The participants were recruited by contacting their organization's occupational health and safety officers. A total of ten organizations participated. Our participants worked in the sectors of telecommunication, banking, and information technology. Participation in this study was anonymous and voluntary. Consent was asked before directed to the questionnaire. The study was approved by the Ethics Review Committee on Human Research, University of Tartu. (Report nr 287/T-26)

\subsection{Study design}

The study was conducted as an online questionnaire. The form consisted of 5 parts: informed consent, gender and age, MSP, self-reported PA, and work environment. The study took place between May and June 2020.

Musculoskeletal complaints were recorded using a modified NORDIC Musculoskeletal Questionnaire. The NORDIC Musculoskeletal Questionnaire records MSP in different body regions with prevalence in the previous 7 days or 6 months. However, in this study, for assessing the impact of lockdown, more detailed options were needed to better identify the onset of MSP. Therefore the answering options for the duration or period of musculoskeletal complaints were modified to (1) no pain, (2) onset 3 months before lockdown, with the pain disappearing during the lockdown, (3) onset during the lockdown, and (4) onset before the lockdown, but the pain is still persistent. When calculating the prevalence of MSP before and during the lockdown, options 2 and 4 sum up as pain before the lockdown and options 3 and 4 as pain during the lockdown.

Self-reported PA was measured using the Baecke Physical Activity Questionnaire (BPAQ). BPAQ has a total of 16 questions and consists of three parts: work-related PA, sport-related PA, and leisure-time PA. The work-related PA section includes questions about the physical demands of work, the sport-related section includes questions about the frequency and intensity of participating in sports, and the leisuretime section about everyday PA outside of work and sports. The answers are used to calculate an index for each section, which sum up as Baecke Physical Activity Index (BPAI). In this study, the questions of BPAQ were duplicated, as first asking retrospectively about 3 months pre-coronavirus period and second during the lockdown. BPAQ has been confirmed as a reliable and valid tool to measure self-reported PA among adults [32].

The work-environment form consisted of questions about work-experience, working with a desktop computer or laptop, frequency of work-at-home days before the lockdown, type of workspace before the lockdown, duration of continuous work without taking a break, and self-reported comfort and ergonomics rating for the workplace. Comfort and ergonomics ratings were collected using a 5-point Likert scale with answering options as (1) strongly disagree, (2) disagree, (3) neither agree or disagree, (4) agree, (5) strongly agree. Comfort rating was asked as "Do you agree, that your work environment and work equipment are comfortable?". Ergonomics rating was inquired as "Do you agree, that your work environment and equipment is ergonomic and adjusted to your personal characteristics to prevent work-related pain?".

\subsection{Statistical analysis}

The data of work environment ratings and selfreported PA indexes are presented as mean $\pm \mathrm{SD}$, with a $95 \%$ confidence interval (CI). A paired $t$-test was used to compare the data of self-reported PA, workplace comfort and ergonomics ratings, and the number of body areas suffering from MSP before and during the lockdown. The effect size was determined using Cohen's d, and the magnitude of the effect is classified as small (from 0.2), medium (from 0.5), and large (from 0.8). A Chi-square test was used to compare the prevalence of MSP in different body areas before and during the lockdown. Pearson correlation analysis was used to find relations between self-reported PA, workplace comfort and ergonomics scores, and MSP. The interpretation of significance in the correlation analysis was calculated as $p<0.05$ on $r>0.155, p<0.01$ on $r>0.203$, and $p<0.001$ on $r>0.257$. The minimal statistical significance level was set to $p<0.05$.

\section{Results}

The prevalence of MSP in different body areas and in total are presented in Fig. 1 There were no 


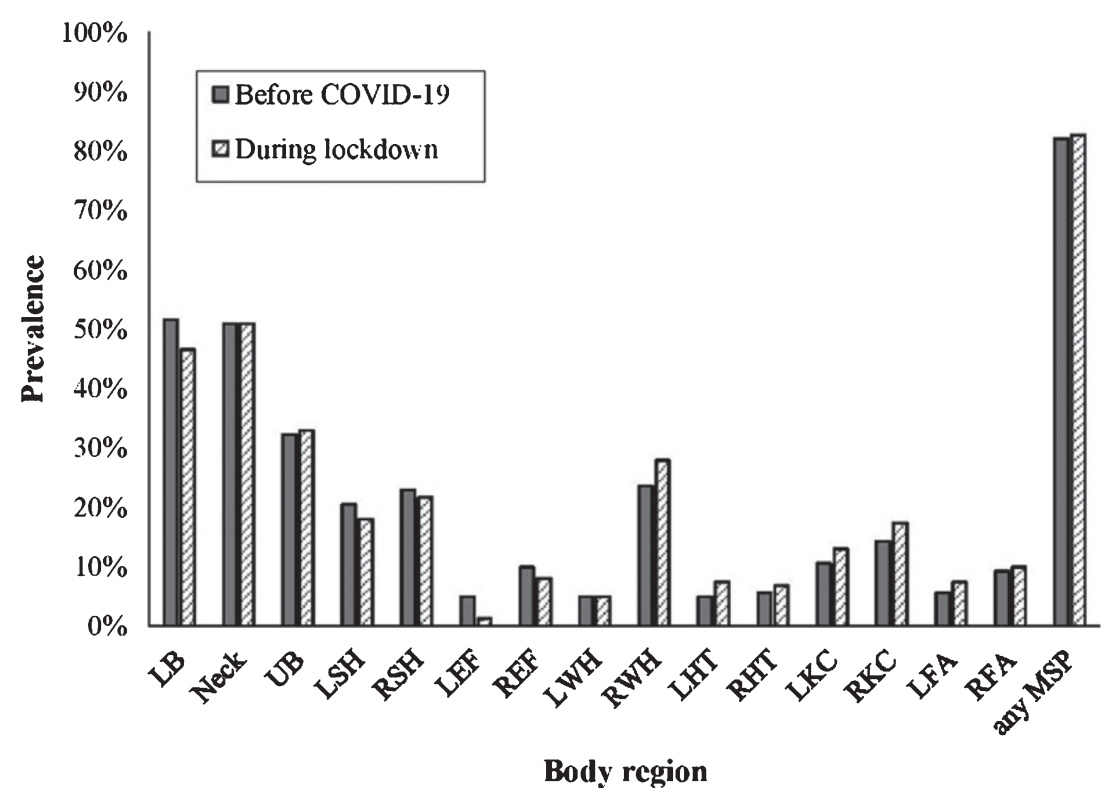

Fig. 1. The prevalence of musculoskeletal pain in office workers $(n=161)$ before and during COVID-19 lockdown in different body regions. Legend: LB - low back, UB - upper back, LSH - left shoulder, RSH - right shoulder, LEF - left elbow \& forearm, REF - right elbow \& forearm, LWH - left wrist \& hand, RWH - right wrist \& hand, LHT - left hip \& thigh, RHT - right hip \& thigh, LKC - left knee \& calf, RKC - right knee \& calf, LFA - left foot \& ankle, RFA - right foot \& ankle, MSP - musculoskeletal pain.

Table 1

Self-reported physical activity of office workers $(n=161)$ before and during COVID-19 lockdown. (mean \pm SD)

\begin{tabular}{lccccc}
\hline $\begin{array}{l}\text { Self-reported } \\
\text { physical activity }\end{array}$ & $\begin{array}{c}\text { Before } \\
\text { COVID-19 }\end{array}$ & $\begin{array}{c}\text { During } \\
\text { lockdown }\end{array}$ & $\begin{array}{c}\text { Change } \\
(95 \% \text { CI })\end{array}$ & $p$ & Cohen's d \\
\hline BPAI work & $2.27 \pm 0.32$ & $2.45 \pm 0.40$ & $+0.18 \pm 0.54(0.10$ to 0.26$)$ & $<0.001$ & $0.50^{\dagger \dagger}$ \\
BPAI sport & $2.94 \pm 1.31$ & $2.42 \pm 1.18$ & $-0.52 \pm 0.98(-0.67$ to -0.37$)$ & $<0.001$ & $0.42^{\dagger}$ \\
BPAI leisure & $2.74 \pm 0.62$ & $2.67 \pm 0.63$ & $-0.07 \pm 0.59(-0.16$ to 0.02$)$ & 0.15 & 0.11 \\
BPAI total & $7.95 \pm 1.60$ & $7.54 \pm 1.55$ & $-0.41 \pm 1.37(-0.62$ to -0.19$)$ & $<0.001$ & $0.26^{\dagger}$ \\
\hline
\end{tabular}

BPAI - Baecke Physical Activity Index; ${ }^{\dagger}$ small effect; ${ }^{\dagger \dagger}$ medium effect.

statistically significant differences in the prevalence of MSP before and during the COVID-19 lockdown in different body areas and in total.

Figure 2 presents the means of body areas with MSP and their chronological features. The mean number of body areas suffering from MSP before and during lockdown was not statistically significant.

During the lockdown, the total self-reported PA was statistically significantly lower $(p<0.001)$ than before the lockdown. (Table 1) The main contributor to lower total self-reported PA was statistically significantly lower $(p<0.001)$ BPAI sports score, however, BPAI work score had statistically significantly increased $(p<0.001)$ during the lockdown. (Table 1) There was no statistically significant change in the BPAI leisure score. (Table 1)

During the lockdown, we noticed an increase $(+25.47 \%)$ in laptop usage for work. Table 2 presents

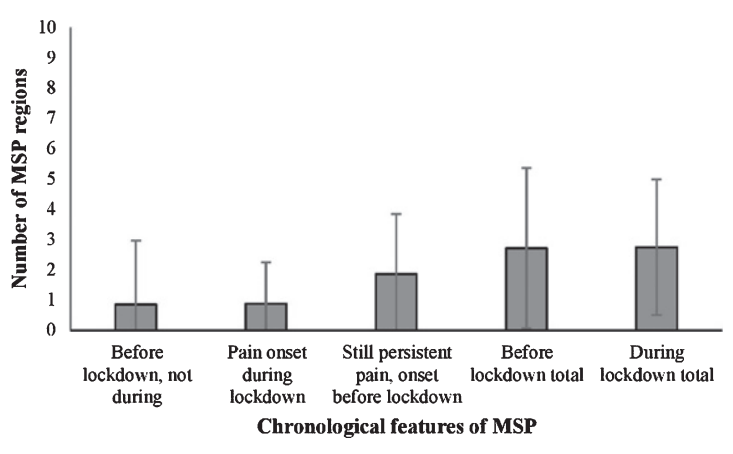

Fig. 2. The mean number of body areas with musculoskeletal pain with different chronological features in office workers. $(n=161)$. Legend: MSP- musculoskeletal pain.

the work environment features before and during COVID-19 lockdown. There was a statistically significant decrease in workplace comfort $(p<0.05)$ and 
Table 2

Features of work environment among office workers before and during COVID-19 lockdown $(n=161)$

\begin{tabular}{lccccc}
\hline Feature & $\begin{array}{c}\text { Before } \\
\text { COVID-19 }\end{array}$ & $\begin{array}{c}\text { During } \\
\text { lockdown }\end{array}$ & $\begin{array}{c}\text { Change } \\
(95 \% \text { CI })\end{array}$ & $p$ & Cohen's d \\
\hline WCR (mean \pm SD) & $3.72 \pm 1$ & $3.44 \pm 1.05$ & $-0.28(-0.51$ to -0.05$)$ & $p<0.05$ & $0.27^{\dagger}$ \\
WER (mean \pm SD) & $4.05 \pm 0.86$ & $3.05 \pm 1.12$ & $-1.00(-1.21$ to -0.79$)$ & $p<0.001$ & $1.00^{\dagger \dagger \dagger}$ \\
HCW (mean \pm SD) & $2.04 \pm 1.05$ & $2.22 \pm 1.30$ & $0.18(-0.01$ to 0.36$)$ & $p=0.06$ & 0.15 \\
\hline
\end{tabular}

WCR - workplace comfort rating; WER - workplace ergonomics rating; HCW - hours of continuous work; ${ }^{\dagger}$ small effect; ${ }^{\dagger \dagger}$ large effect.

workplace ergonomics rating $(p<0.001)$. The hours of continuous work did not increase statistically significantly $(p=0.06)$.

There was a significant negative correlation $(p<0.01)$ between the change in self-reported sports PA and the change in the numbers of body regions with MSP during the lockdown (Fig. 3a). A significant negative correlation $(p<0.001)$ was present between the change in workplace comfort score and the number of body regions with MSP onset during the lockdown (Fig. 3b). We also found a negative significant correlation $(p<0.01)$ between the change in workplace ergonomics score and the number of body regions with MSP onset during the lockdown (Fig. 3c).

\section{Discussion}

This study indicated no significant differences in the prevalence of overall MSP and MSP in specific body regions before and during the lockdown. In light of recent findings of the lockdown's negative impact on mental well-being, such as worsening of anxiety, depression, and stress [33], our result was surprising. Since MSP is a biopsychosocial phenomenon $[34,35]$ and previous evidence, that the home-office population might experience an increase in mental health issues and pain affecting the spine [13], we expected an increase in the prevalence of MSP during the lockdown. Both not measuring the intensity of pain and investigating mental health issues we consider a limitation of our study and we are not certain how the lockdown affected pain intensity among our subjects. However, we consider the 66 days of exposure to the lockdown as not enough to cause changes in the prevalence of MSP.

Since PA has an important effect on the prevention and treatment of MSP [19, 20, 36, 37], sudden changes can have an impact on MSP. In this study, we hypothesized, that due to the closing of sports facilities, people compensate for their indoor work-
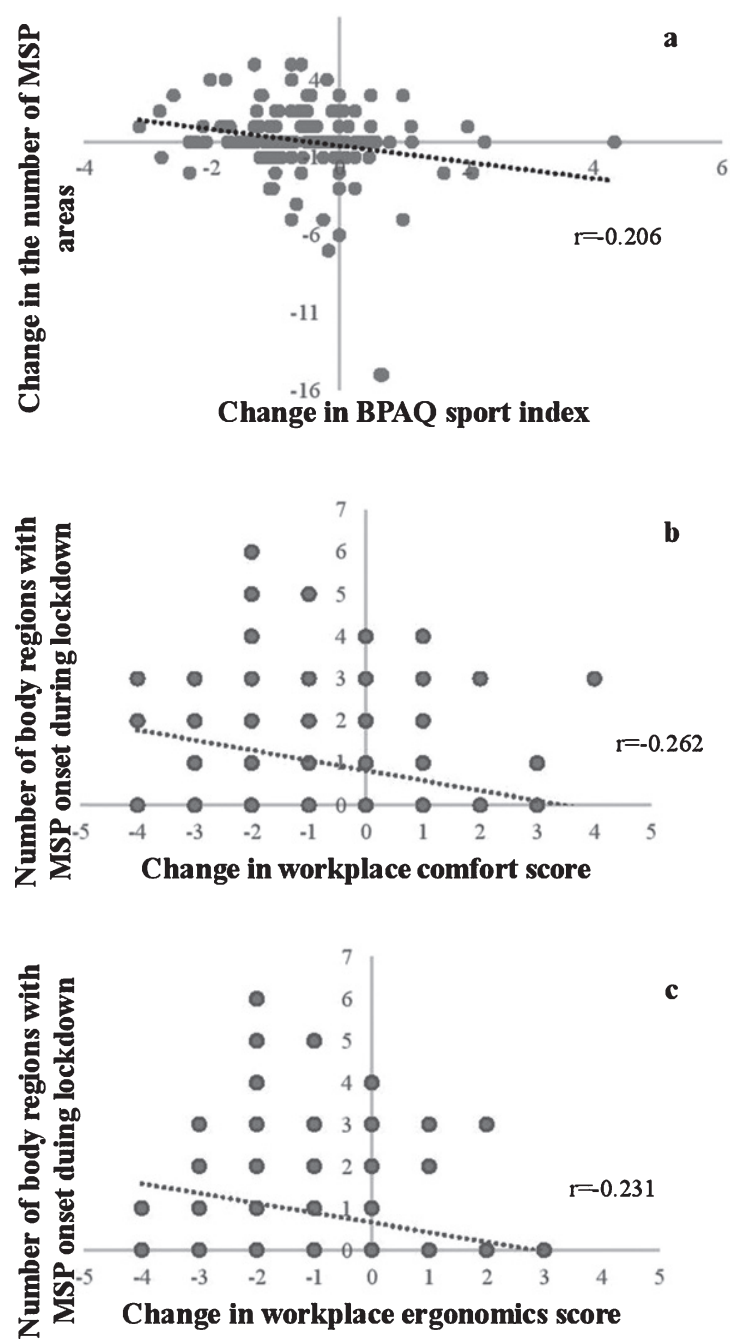

Fig. 3. Correlations between the change in self-reported sportrelated physical activity and the change in the number of body regions with musculoskeletal pain, between the change in workplace comfort and ergonomics scores and musculoskeletal pain onset during the lockdown. $(n=161)$. Legend: MSP - musculoskeletal pain. BPAQ - Baecke Physical Activity Questionnaire.

outs by engaging in more leisure-time PA. We also hypothesized an increase in work-related PA due to a more flexible schedule and a smaller boundary 
between work, family, and home-related tasks. The results indicate that our hypothesis became partly true, however, the effect size was small. Our result of an overall decrease in self-reported PA among office workers is similar to other populations studied during the COVID-19 lockdown period [24, 31]. However previous studies haven't investigated the subclasses of PA. When looking at the overall participation in sports, it remained similar (78\% before and $81 \%$ during the lockdown). However, our secondary analysis of independent questions from the BPAQ sports section showed several reasons behind the lower BPAI sports score. First appeared to be lower participation in more than one sport activity. $96 \%$ of participants who engaged in exercising regularly used to have more than one sport activity before the lockdown. During the lockdown having more than one sport activity decreased to $62 \%$ of participants. The second reason appeared to be significantly $(p<0.001)$ lower sport intensity and frequency, which means a lot of participants switched their activity for another, with fewer training sessions per week and less intensity in nature.

The reason behind significantly higher workrelated PA appeared to be a significantly lower sedentary time and more time spent standing during work, while other physical activities during work, such as walking or lifting heavy objects appeared to be lower when working from home during the lockdown. Less sedentary time and less walking might be controversial, but when answering the questionnaire, the participants might not have reasoned taking a few steps while participating in home and family-related tasks as walking. On the other hand, walking to a colleague in the office or going for lunch might be described as walking since it has a longer duration when compared with taking a few steps at home. Since we found no significant difference in the duration of continuous work, and there was less sedentary and more standing time, the participants could have worked more in other positions than sitting. Also, our participants might not have counted interruptions at home as taking a break, since no difference in continuous work duration. We did not include questions about family in this study, such as the number and age of children. Since the mean age of our sample was 38.2 years, we can assume many of them have school-aged children who also had to study from home. Therefore, parents might have overlapped responsibilities of working from home and minding their children at the same time [7]. Mixing work with such responsibilities might lead to less continuous computer use and slightly more PA during the working hours, but at the same time to more psychosocial strain.

The correlation analysis suggested that a greater decrease in sport-related PA score is related to an increase in the number of body regions suffering from MSP. Therefore, such sudden negative changes in sport-related PA might increase the sensibility to MSP. Since we did not include the measurement of pain intensity in our study, we are not certain how this kind of sudden decrease in habitual PA can influence already existing MSP. There are physical and psychosocial factors that could contribute to this finding. One of the reasons exercising or exercise therapy is considered an effective prevention and treatment method of MSP is due to its hypoalgesic properties $[38,39]$. The decrease in the intensity and frequency of exercise could contribute to increased sensitization to noxious stimuli, as less exercise might cause less exercise-induced hypoalgesia, therefore more sensitivity. Since engaging in sports activities is often also a social occasion, the loss of exercising with friends could contribute to psychosocial issues related to social isolation such as loneliness, stress, or depression, which are associated with MSP [40, 41]. There have been similar findings in people suffering from chronic pain during the lockdown period, as decreases in PA lead to increased perception of pain [31]. Considering our findings and previous evidence, exercise can be an important method in MSP prevention.

Because to the switch to working from home every day had been sudden, the decreases in workplace comfort and ergonomics scores were expected. This finding suggests that for most participants, comfort and ergonomics are interchangeable terms. One of the reasons for this decrease could be the switch from working with a desktop computer to a less comfortable laptop computer. In this study, the number of laptop users increased by $25.5 \%$ during the lockdown. Using a laptop instead of a desktop computer can lead to working in more awkward positions and in places not designed for everyday working. There has also been found a link between laptop usage and the worsening of neck pain during the lockdown [13]. We found significant negative correlations, as participants with a greater decrease in workplace comfort and ergonomics score had more MSP with an onset during the lockdown. This correlation indicates that improving the comfort and ergonomics of the workplace might help to prevent MSP. Therefore, the employees who continue to work from home need better working conditions and more support, since 
Montreuil and Lippel's trial concluded that adequate equipment and ergonomic support might contribute to even higher-rated working conditions at home when compared with office workspace [5]. Considering the results, more attention to workplace comfort and ergonomics is needed at home to prevent MSP.

The main limitations of this study were the questionnaire-based design, retrospective questions, and the absence of data about psychosocial factors and pain intensity. Although BPAQ is considered a valid and reliable questionnaire for measuring selfreported PA [32], it has several limitations. The main limitation is the questionnaire not considering the energy-expenditure or nature of activities in the separate sections. This makes work score, sports score, and leisure score not comparable with each other, and therefore the validity of the total score questionable, which is the reason we decided to analyze the scores separately. Since we asked the questions twice, first retrospectively about 3 months before the lockdown and secondly at the moment of answering, during the lockdown, the participants might have had a bias towards more negative answers about the lockdown period. Because the questions about the period before the lockdown were retrospective with several months in between, recall bias should also be considered. Since data about the health factors and ergonomics of the home-office population is still scarce, further research should investigate the home-office population with more detailed measurements, such as pedometry and position sensors. Future research could also incorporate interviews about the work environment at home, occupational health, and health beliefs for a better understanding of the risks and benefits of teleworking. For further conclusions, replication studies in non-lockdown settings are needed to better distinguish between lockdown-related and non-lockdown-related factors contributing to the musculoskeletal health of the home-office population.

\section{Conclusion}

In summary, the lockdown period of 66 days in Estonia, which caused office workers to work from home, did not change the prevalence or the mean amount of body regions with MSP among office workers in this study. There was a decrease in overall BPAI and sport-related PA scores and an increase in work-related PA scores during the lockdown when compared with a period of up to 3 months before.
The main reason for lower PA and sport-related PA was a change to less intense and less frequent exercise, while the overall participation in sports remained similar. The increase in work-related PA score occurred mostly from less sedentary time and more time spent standing. The sudden change to working from home caused a decrease in workplace comfort and ergonomics scores. Our analysis suggests, that on an individual level, a larger decrease in sport-related PA can be associated with MSP in more body regions. Also, lower comfort and ergonomics scores were associated with a higher chance of developing MSP during the lockdown period. In addition to physical and workplace factors, future studies about teleworkers' health should also investigate the MSPrelated psychosocial factors. Maintaining the habitual PA level and preparing the home environment for more comfortable and ergonomic working can play an important role in a healthier transition to working from home.

\section{Acknowledgments}

We would like to thank all the participants for taking the time and contributing their data. This research received no specific funding from any funding agency in the public, commercial, or not-for-profit sectors.

\section{Author contributions}

MA conceived the idea, designed the study, collected and analyzed the data, drafted the manuscript. MP advised on the methods and structuring the draft, revised the manuscript. Both authors read and approved the final manuscript.

\section{Conflict of interest}

The authors declare that there is no conflict of interest to report.

\section{References}

[1] World Health Organisation. 2020. WHO Timeline - Covid19. Available online: https://www.who.int/news/item/2704-2020-who-timeline-covid-19 (accessed on 1. Nov 2020).

[2] Republic of Estonia, Health Board. 2020. Available online: https://www.kriis.ee/en/coronavirus-and-healthcare (accessed on 1. Nov 2020). 
[3] Savić D. COVID-19 and Work from Home: Digital Transformation of the Workforce. The Grey Journal. 2020;16(2): 101-4.

[4] Harpaz I. Advantages and Disadvantages of Telecommuting for the Individual, Organization, and Society. Work Study. 2002;51(2):74-80. doi: 10.1108/00438020210418791

[5] Montreuil S, Lippel K. Telework and occupational health: a Quebec empirical study and regulatory implications. Safety Science. 2003;41:339-58.

[6] de Macêdo TAM, et al. Ergonomics and Telework: A Systematic Review. WORK. 2020;66(4):777-88. Doi: 10.3233/ WOR-203224

[7] Bouziri H, Smith DRM, Descatha A, Dab W, Jean K. Working from home in the time of covid-19: how to best preserve occupational health? Occup Environ Med. 2020;77(7):50910. doi: 10.1136/oemed-2020-106599

[8] Kaliniene G, Ustinaviciene R, Skemiene L, Vaiciulis V, Vasilavicius P. Associations between musculoskeletal pain and work-related factors among public service sector computer workers in Kaunas County, Lithuania. BMC Musculoskelet Disord. 2016;17(1):420. doi: 10.1186/s12891016-1281-7

[9] Shariat A, Bahri S, Tamrin SBM, Arumugam M. Musculoskeletal disorders and their relationship with physical activities among office workers: a review. Malaysian J Public Health Med. 2016;16:62-74.

[10] Wu S, He L, Li J, Wang J, Wang S. Visual display terminal use increases the prevalence and risk of work-related musculoskeletal disorders among Chinese office workers: a cross-sectional study. J Occup Health. 2012;54:34-43.

[11] da Costa BR, Vieira ER. Risk factors for work-related musculoskeletal disorders: a systematic review of recent longitudinal studies. Am J Ind Med. 2010;53:285-323.

[12] Celenay ST, Karaaslan Y, Mete O, Kaya DO. Coronaphobia, musculoskeletal pain, and sleep quality in stay-at-home and continued-working persons during the 3-month Covid-19 pandemic lockdown in Turkey. Chronobiology International. 2020. doi: 10.1080/07420528.2020.1815759

[13] Moretti A, Menna F, Aulicino M, Paoletta M, Liguori $\mathrm{S}$, Iolascon G. Characterization of HomeWorking Population during COVID-19 Emergency: A Cross-Sectional Analysis. J Environ Res Public Health. 2020;17:6284. doi: 10.3390/ijerph17176284

[14] Holth HS, Werpen HK, Zwart JA, Hagen K. Physical inactivity is associated with chronic musculoskeletal complaints 11 years later: results from the Nord-Trøndelag Health Study. BMC Musculoskelet Disord. 2008;9:159. doi: 10.1186/1471-2474-9-159.

[15] Lippi G, Henry BM, Sanchis-Gomar F. Physical inactivity and cardiovascular disease at the time of coronavirus disease 2019 (COVID-19). Eur J Prev Cardiol. 2020;27(9):906-8. doi: 10.1177/2047487320916823

[16] Thorp AA, Owen N, Neuhaus M, Dunstan DW. Sedentary behaviors and subsequent health outcomes in adults a systematic review of longitudinal studies. Am J Prev Med. 2011;41:207-15.

[17] Proper KI, Singh AS, van Mechelen W, Chinapaw MJ. Sedentary behaviors and health outcomes among adults: a systematic review of prospective studies. Am J Prev Med. 2011;40:174-82.

[18] Biswas A, Oh PI, Faulkner GE, Bajaj RR, Silver MA, Mitchell MS, Alter DA. Sedentary time and its association with risk for disease incidence, mortality, and hospitalization in adults: a systematic review and meta-analysis. Ann Intern Med. 2015;162(2):123-32. doi: 10.7326/M14-1651.
[19] Blangsted AK, Søgaard K, Hansen EA, Hannerz H, Sjøgaard G. One-year randomized controlled trial with different physical-activity programs to reduce musculoskeletal symptoms in the neck and shoulders among office workers. Scand J Work Environ Health. 2008;34(1):55-65. doi:10.5271/sjweh.1192

[20] Moreira-Silva I, Teixeira PM, Santos R, Abreu S, Moreira C, Mota J. The Effects of Workplace Physical Activity Programs on Musculoskeletal Pain: A Systematic Review and Meta-Analysis. Workplace Health Saf. 2016;64:210-22. doi: $10.1177 / 2165079916629688$.

[21] Martinez M, Latorre M. Health and work ability among office workers. Revista de saúde pública. 2006;40:851-8.

[22] Mikkelsen K, Stojanovska L, Polenakovic M, Bosevski M, Apostolopoulos V. Exercise and mental health. Maturitas. 2017;106:48-56. doi:10.1016/j.maturitas.2017.09.003

[23] Pieh, C, Budimir S, Delgadillo J, Barkham, M, Fontaine, JR, Probst T. Mental health during COVID-19 lockdown in the United Kingdom. Psychosomatic Medicine. 2020 Volume Publish Ahead of Print. doi: 10.1097/PSY.00000 00000000871

[24] Bourdas DI, Zacharakis ED. Evolution of changes in physical activity over lockdown time: Physical activity datasets of four independent adult sample groups corresponding to each of the last four of the six COVID-19 lockdown weeks in Greece. Data in Brief. 2020;32:106310. doi: 10.1016/j.dib.2020.106301

[25] Knardahl S. Psychophysiological mechanisms of pain in computer work: The blood vessel-nociceptor interaction hypothesis. Work Stress. 2002;16:179-89. doi: 10.1080/ 02678370210140117.

[26] Hägg G. Static work load and occupational myalgia - A new explanation model. Electromyographical kinesiology. 1991:141-4

[27] Wahlström J. Ergonomics, musculoskeletal disorders, and computer work. Occup med. 2005;55:168-76. doi: 10.1093/ occmed/kqi083.

[28] Korhonen T, Ketola R, Toivonen R, Luukkonen R, Häkkänen M, Viikari-Juntura E. Work-related and individual predictors for incident neck pain among office employees working with video display units. Occup Environ Med. 2003;60(7):475-82. doi: 10.1136/oem.60.7.475.

[29] van Niekerk S, Louw QA, Hillier S. The effectiveness of a chair intervention in the workplace to reduce musculoskeletal symptoms. A systematic review. BMC Musculoskeletal Disorders. 2012;13(1):145. doi: 10.1186/1471-247413-145

[30] Agarwal S, SteinmausC, Harris-Adamson C. Sit-stand workstations and impact on low back discomfort: a systematic review and meta-analysis. Ergonomics. 2018;61(4): 538-552, doi: 10.1080/00140139.2017.1402960

[31] Fallon N, Brown C, Twiddy H, Brian E, Frank B, Nurmikko T, Stancak A. Adverse effects of COVID-19 related lockdown on pain, physical activity and psychological wellbeing in people with chronic pain. medRxiv. 2020;06.04.20122564. doi: 10.1101/2020.06.04.20122564

[32] Ono R, Soichiro H, Minoru Y, Takayuki N, Masahiro K, Yumi T. Reliability and validity of the Baecke physical activity questionnaire in adult women with hip disorders. BMC Musculoskslet Disord. 2007;8:61. doi: 10.1186/1471-2474$8-61$.

[33] Torales J, O’Higgins M, Castaldelli-Maia JM, Ventriglio A. The outbreak of COVID-19 coronavirus and its impact on global mental health. Int J Soc Psychiatry. 2020;66(4):31720. doi: $10.1177 / 0020764020915212$ 
[34] Vargas-Prada S, Coggon D. Psychological and psychosocial determinants of musculoskeletal pain and associated disability. Best Pract Res Clin Rheumatol. 2015;29(3):374-90. doi: 10.1016/j.berh.2015.03.003.

[35] Gatchel RJ, Peng YB, Peters ML, Fuchs PN, Turk DC. The biopsychosocial approach to chronic pain: Scientific advances and future directions. Psychological Bulletin. 2007;133(4):581-624.

[36] Nijs J, Lluch Girbés E, Lundberg M, Malfliet A, Sterling M. Exercise therapy for chronic musculoskeletal pain: Innovation by altering pain memories. Man Ther. 2015;20(1):216-20. doi: 10.1016/j.math.2014.07.004.

[37] Rodrigues EV, Gomes AR, Tanhoffer AI, Leite N. Effects of exercise on pain of musculoskeletal disorders: a systematic review. Acta Ortop Bras. 2014;22(6):334-8. doi: 10.1590/1413-78522014220601004.
[38] Drury D, Greenwood K, Stuempfle K, Koltyn K. Changes in Pain Perception in Women During and Following an Exhaustive Incremental Cycling Exercise. J Aports Sci Med. 2005;4:215-22.

[39] Koltyn KF, Brellenthin AG, Cook DB, Sehgal N, Hillard C. Mechanisms of exercise-induced hypoalgesia. J Pain. 2014;15(12):1294-304. doi:10.1016/j.jpain.2014.09.006

[40] Ng YM, Voo P, Maakip I. Psychosocial factors, depression, and musculoskeletal disorders among teachers. BMC Public Health. 2019;234. doi: 10.1186/s12889-019-6553-3

[41] Smith TO, Dainty JR, Williamson E, Martin KR. Association between musculoskeletal pain with social isolation and loneliness: analysis of the English Longitudinal Study of Ageing. Br J Pain. 2019;13(2):82-90. doi:10.1177/ 2049463718802868 